\title{
Approximation of fixed points for nonexpansive operators by means of the fastest Krasnoselskij iteration in Hilbert spaces
}

\author{
Toseef Ahmed Malik ${ }^{1}$ and Masood Ahmed Choudhary ${ }^{2}$ \\ ${ }^{1}$ Department of Mathematics, University of Jammu, Jammu, India \\ ${ }^{2}$ Department of Mathematics, Govt. Hr. Sec. School Mohore, Reasi, J and K, India
}

Received: 5 May 2018, Accepted: 27 June 2018

Published online: 23 May 2019.

\begin{abstract}
The interest of this paper is to survey some old and new results on the approximation of fixed points for nonexpansive and pseudocontractive type operators by means of the fastest Krasnoselskij iteration. We consider here generalized pseudocontractions which are also Lipschitzian, a class for which we can use the Krasnoselskij iteration in order to approximate their fixed points.
\end{abstract}

Keywords: Demicompact, fixed point, generalized pseudo-contraction, Hilbert space, Krasnoselskij iteration, nonexpansive.

\section{Introduction}

In the last three decades many papers have been published on the iterative approximation of fixed point for certain classes of operators, using the Mann and Ishikawa iteration methods, see for example Berinde [4], for recent survey. These papers were motivated by the fact that, under weaker contractive type condition, the Picard iteration (or the method of successive approximation), $x_{n+1}=T x_{n}, n \geq 0$ need not converge to the fixed point of the operators in question.

In order to approximation fixed points of certain classes of operators which satisfy weak contractive type conditions that do not guarantee the convergence of Picard iterative process (or the method of successive approximation), certain mean value fixed point iterations, namely Krasnoselskij, Mann and Ishikawa iteration methods are useful to approximate fixed points. Though these iterative procedures have been introduced mainly in order to approximate fixed points of those operators for which the Picard iteration does not converge, even so there are results on important class of contractive mappings, that is, the class of quasi-contraction, for which all Picard, Krasnoselskij, Mann and Ishikawa iterations converge. One of the most studied class of quasi-contractive type operators is that of Zamfirescu operators, for which all important fixed point iteration procedures, i.e, the Picard, Mann and Ishikawa iterations, are known to converge to the unique fixed point of the operator $T$. Zamfirescu showed in [10 ] that an operator which satisfies the contractive conditions has a unique fixed point that can be approximated using the Picard iteration. Later, Rhoades [8], proved that the Mann and Ishikawa iterations can also be used to approximate fixed points of Zamfirescu operators.

Verma [9] approximated fixed points of strictly pseudo-contractive operators in Hilbert spaces by both Krasnoselskij and Mann type iterative methods. When for a certain class of mappings, two or more fixed points iteration procedures can be used to approximate their fixed points, it is of theoretical and practical importance to compare the rate of convergence of these methods and to find out, if possible which of them converges faster. Finally, we shall obtain a result on the fastest 
iteration in the family of the krasnoselskij iterative scheme. As far as we known, there are only a few papers devoted to this very important numerical problem. It is also well known that if $T$ is assumed to be only a nonexpasive map, then the Picard iteration $\left\{T^{n} x_{0}\right\}_{n \geq 0}$ need no longer converge. The key idea in introducing Krasnoselskij iteration is that fact that, if $T_{\lambda}$ is the averaged mapping associated to $T$, then if $T$ is nonexpasive, so is $T_{\lambda}$, and both have the same fixed point set. Moreover, $T_{\lambda}$ has much more asymptotic behavior than the original mapping $T$.

\section{Preliminaries}

Definition 1. Let $H$ be a real vector space. An inner product is a functional $\langle\cdot, \cdot\rangle: H \times H \rightarrow \mathbb{R}$ (the set of real numbers) satisfying:

(p1) $\langle x, x\rangle \geq 0$, for all $x, y \in H$;

(p2) $\langle x, y\rangle=\langle y, x\rangle$, for all $x, y \in H$;

(p3) $\langle\alpha x+\beta y, z\rangle=\alpha\langle x, z\rangle+\beta\langle y, z\rangle$, for each $x, y, z \in H$ and all $\alpha, \beta \in \mathbb{R}$.

If $\langle\cdot, \cdot\rangle$ is an inner product on $H$, then the function $x \rightarrow\langle x, x\rangle^{1 / 2}$ defines a norm on $H$, called the norm induced by the inner product. The pair $(H,\langle\cdot, \cdot\rangle)$ is called a prehilbertian space.

A sequence $\left\{x_{n}\right\}$ in $H$ is called Cauchy sequence iff $\left\langle x_{n}-x_{m}, x_{n}-x_{m}\right\rangle^{1 / 2}=\left\|x_{n}-x_{m}\right\| \rightarrow 0$ as $n, m \rightarrow 0$. A prehilbertian space $H$ is said to be complete if every Cauchy sequence in $H$ converges to a point of $H$. A prehilbertian space that is complete (with respect to the metric corresponding to the norm induced by the scalar product) is called Hilbert space.

Definition 2. Let $H$ be a real Hilbert space with $\|\cdot\|$ and an inner product $\langle\cdot, \cdot\rangle$. An operator $T: H \rightarrow H$ is said to be generalized pseudo-contraction if, for all $x, y$ in $H$, there exists a constant $r>0$ such that

$$
\|T x-T y\|^{2} \leq r^{2}\|x-y\|^{2}+\|T x-T y-r(x-y)\|^{2}
$$

which is equivalent to

$$
\begin{gathered}
\langle T x-T y, x-y\rangle \leq r\|x-y\|^{2}, \text { for all } x, y \in H \\
\text { or to }\langle(I-T) x-(I-T) y, x-y\rangle \geq(1-r)\|x-y\|^{2},
\end{gathered}
$$

where I is the identity map. Clearly, if $T$ is generalized pseudo-contraction with $r<1$, then I-T is strongly monotone. For $r=1$ in (2.1), $T$ is called pseudo-contraction, a concept introduced and studied by Browder and Petryshyn [6] and thereafter by many authors, in connection with the problem of approximating fixed points, see Berinde [4].

Definition 3. The operator $T: H \rightarrow H$ is called Lipschitzian (or Lipschitz continuous) if there exist a constant $s>0$ such that

$$
\|T x-T y\| \leq s \cdot\|x-y\|, \text { for all } x, y \in H .
$$

By the Cauchy Schwarz inequality

$$
|\langle T x-T y, x-y\rangle| \leq\|T x-T y\|\|x-y\| .
$$

It is clear that any Lipschitzian operator $T$, that is, any operator for which there exists $s>0$ such that $\|T x-T y\| \leq s\|x-y\|, x, y \in H$ is also a generalized pseudo-contractive operator, with $r=s$ and $T$ is non-expansive if $T$ is 1 - Lipschitzian. 
Example 1. [4] Let $H$ be the real line $\mathbb{R}$ endowed with the Euclidean inner product and norm, $C=\left[\frac{1}{2}, 2\right]$ and $T: C \rightarrow C$ a self map defined by $T x=\frac{1}{x}$, for all $x \in C$. Then $T$ is Lipschitzian with constant $s=4$ and also generalized pseudocontractive with constant $r=4$. Moreover, $T$ is also generalized pseudo-contractive with any constant $r>0$ arbitrary.It is easy to see that $T$ has a unique fixed point, $F_{T}=\{1\}$, and that, for any initial choice $x_{0}=a \neq 1$, the Picard iteration yields the oscillatory sequence $a, 1 / a, a, 1 / a, \ldots$.

Definition 4. A subset $C$ of a real vector space $H$ is called convex if for any pairs of points $x, y \in C$, the closed segment with the extremities $x, y$, that is, the set $\{\lambda x+(1-\lambda) y: \lambda \in[0,1]$ is contained in $C$. A subset $C$ of $H$ is called bounded if there exists $M>0$ such that $\|x\| \leq M$, for all $x \in C$.

Definition 5. Let $H^{*}$ be the dual space of a real Hilbert space. The multivalued mapping $J: H \rightarrow P\left(H^{*}\right)$ defined by $J x=\left\{f \in H^{*}:\langle f, x\rangle=\|x\| \cdot\|f\|,\|x\|=\|f\|\right\}$ is called the normalized duality mapping of $H$.

In Hilbert space $H$, a sequence $\left\{x_{n}\right\}_{n=0}^{\infty} \subset H$ converges strongly to $x$ if and only if $\left\|x_{n}-x\right\| \rightarrow 0$, as $n \rightarrow \infty$ which is denoted by $x_{n} \rightarrow x$, we shall often consider the weak convergence, corresponding to the weak topology in $H$. We say that $\left\{x_{n}\right\}_{n=0}^{\infty} \subset H$ converges weakly to $x$ if for any $f \in H^{*},\left\langle f, x_{n}\right\rangle \rightarrow\langle f, x\rangle$, as $n \rightarrow \infty$ and we denote this by $x_{n} \rightarrow x(n \rightarrow \infty)$.

Since conditions of pseudo-contractive type are very useful additional assumptions in approximating fixed points of Lipschitzian mappings, we summarize in the sequel the most important concepts of this kind.

Definition 6. Let $H$ be an arbitrary Hilbert space. A mapping $T$ with domain $D(T)$ and range $R(T)$ in $H$ is called:

(a) strongly pseudocontraction if there exists $k>0$ such that for all $x, y \in D(T)$ there exists $j(x, y) \in J(x-y)$ such that

$$
\langle(I-T) x-(I-T) y, j(x-y)\rangle \geq k \cdot\|x-y\|^{2}
$$

(b) pseudocontractive if for each $x, y \in D(T)$ there exists $j(x-y) \in J(x-y)$ such that

$$
\langle(I-T) x-(I-T) y, j(x-y)\rangle \geq 0,
$$

where $J$ is the normalized duality mapping.

Pseudo-contractive mappings are firmly connected with another important class of operators, that is, the class of accretive operators.

Definition 7. A mapping $T$ with domain and range in $H$ is called:

(a) strongly accretive if there exists a positive number $k$ such that for each $x, y \in D(T)$ there is a $j(x-y) \in J(x-y)$ such that

$$
\langle T x-T y, j(x-y)\rangle \geq k \cdot\|x-y\|^{2}
$$

(b) accretive if for each $x, y \in D(T)$ we have

$$
\langle T x-T y, j(x-y)\rangle \geq 0 .
$$

Remark.

(i) By comparing Definitions (2.7) and (2.8), we remark that an operator $T$ is (strongly) pseudo-contractive if and only if $(I-T)$ is (strongly) accretive. 
(ii) As a consequence of a result of Kato [1], the concept of pseudo-contractive and accretive operators can be equivalently defined as follows:

(a) $T$ is strongly pseudo-contractive if there exists $t>1$ such that, for all $x, y \in D(T)$ and $r>0$, the following inequality holds

$$
\|x-y\| \geq\|(1+r)(x+y)-r t(T x-T y)\|
$$

(b) $T$ Is pseudo-contractive if $t=1$ in the previous inequality.

(c) $T$ is strongly accretive if there exists $k>0$ such that the inequality

$$
\|x-y\| \leq\|x-y+r[(T-k I) x-(T-k I) y]\|
$$

holds for all $x, y \in D(T)$ and $r>0$.

(d) $T$ is accretive if $k=0$ in in the previous inequality.

\section{Some fixed point iteration procedures:}

Let $T: H \rightarrow H$ be a self map. For any $x_{0} \in H$, the sequence $\left\{x_{n}\right\}_{n=0}^{\infty} \subset H$ given by

$$
x_{n}=T x_{n+1}=T^{n}\left(x_{0}\right), \quad n=1,2 \ldots \ldots
$$

is called the Picard iteration or the sequence of successive approximations with the initial value at $x_{0}$.

Let $H$ be a Hilbert space and $T: H \rightarrow H$ a self map. For any $x_{0} \in H$ and a constant $\lambda \in[0,1]$ the sequence $\left\{x_{n}\right\}_{n=0}^{\infty}$ defined by

$$
x_{n+1}=(1-\lambda) x_{n}+\lambda T x_{n}, \quad n=0,1,2 \ldots \ldots
$$

is called Krasnoselskij iteration or Krasnoselskij iterative method and is denoted by $K_{n}\left(x_{0}, \lambda, T\right)$.

Let $H$ be a Hilbert space and $T: H \rightarrow H$ a self map. Let $x_{0} \in H$ be arbitrary and $\left\{\alpha_{n}\right\}_{n=0}^{\infty} \subset[0,1]$ a sequence of real numbers. The sequence $\left\{x_{n}\right\}_{n=0}^{\infty} \subset H$ defined by

$$
x_{n+1}=\left(1-\alpha_{n}\right) x_{n}+\alpha_{n} T x_{n}, \quad n=0,1,2 \ldots \ldots
$$

is called the Mann iteration or Mann iterative procedure and will be denoted by $M_{n}\left(x_{0}, \alpha_{n}, T\right)$. The sequence $\left\{x_{n}\right\}_{n=0}^{\infty} \subset$ $H$ defined by

$$
\begin{aligned}
x_{n+1} & =\left(1-\alpha_{n}\right) x_{n}+\alpha_{n} T y_{n}, \quad n=0,1,2 \ldots \ldots \\
y_{n} & =\left(1-\beta_{n}\right) x_{n}+\beta_{n} T x_{n}, \quad n=0,1,2 \ldots \ldots \ldots \ldots
\end{aligned}
$$

where $\left\{\alpha_{n}\right\}_{n=0}^{\infty}$ and $\left\{\beta_{n}\right\}_{n=0}^{\infty}$ are sequences of positive numbers in $[0,1]$, and $x_{0} \in H$ is arbitrary, is called the Ishikawa iteration or Ishikawa iterative procedure and is denoted by $I\left(x_{0}, \alpha_{n}, \beta_{n}, T\right)$.

Remark. For $\alpha_{n}=\lambda$ (constant), the iteration (3.3) reduces to the so called Krasnoselskij iteration while for $\alpha_{n}=1$ we obtain the Picard iteration or the sequence of successive approximation (3.1), as it is commonly known obviously, for $\beta_{n}=0$, the Ishikawa iteration (3.4) reduces to Mann iteration (3.3).

Example 2. Let $C=\left[\frac{1}{2}, 2\right] \subseteq \mathbb{R}$ be endowed with the usual norm and $T: C \rightarrow C$, defined by $T x=\frac{1}{x}, x \in C$. Then 
(i) $T$ has a unique fixed point, that is $F_{T}=\{1\}$;

(ii) The Picard iteration does not converge 1 , for any $x_{0} \neq 1$ in $\left[\frac{1}{2}, 2\right]$;

(iii) The Krasnoselskij iteration converges to the fixed point of $T$, for $\lambda$ satisfying $0<\lambda<2(1-r) /(17-2 r)$, where $0<r<1$.

The convergence theorems below, stated for the Krasnoselskij and Mann iterative procedures respectively, by Verma [9] will be used in the proof of the main results.

Theorem 1. [2] Let $C$ be a non-empty closed convex subset of a real Hilbert space $H$ and $T: C \rightarrow C$ a generalized pseudo-contractive and Lipschitzian operator with the corresponding constants $s>0$ and $r>0$ satisfying

$$
0<r<1, \quad r \leq s
$$

Then

(i) T has a unique fixed point p;

(ii) For each $x_{0}$ in $C$, the Krasnoselskij iteration $\left\{x_{n}\right\}_{n=0}^{\infty}$ given by $x_{n+1}=(1-\lambda) x_{n}+\lambda T x_{n}, \quad n=0.1,2 \ldots$. converges strongly to $p$, for all $\lambda \in(0, a) \cap(0,1)$,

$$
\text { where } a=2(1-r) /\left(1-2 r+s^{2}\right)
$$

Theorem 2. [9] Let $H$ be a real Hilbert space and $C$ be a non-empty closed convex subset of $H$. Let $T: C \rightarrow C$ be Lipschitzian and generalized pseudo-contractive operator with the corresponding constants $s>1$ and $r>0$. Let $\left\{\alpha_{n}\right\}_{n=0}^{\infty}$ be an increasing sequence in $[0,1]$ such that

$$
\sum_{n=0}^{\infty} \alpha_{n}=\infty
$$

Then

(i) $T$ has a unique fixed point $p$ in $C$;

(ii) The Mann iteration $x_{n+1}=\left(1-\alpha_{n}\right) x_{n}+\alpha_{n} T x_{n}, \quad n=0,1,2 \ldots$ converges strongly to $p$ for any $x_{0} \in C$ and all $t$ in $(0, a)$ that satisfy $0 \leq(1-t)^{2}-2 t(1-t) r+t^{2} s^{2}<1$, where a is given by (3.6).

Example 3. Let $C=\left[\frac{1}{2}, 2\right]$ and $T: C \rightarrow C$ a function given by $T x=\frac{1}{x}, x \in C$. Here $s=4$ and $r>0$ arbitrary. Taking, for example $r=0.5$, we get $2(1-r) /\left(1-2 r+s^{2}\right)=\frac{1}{16}$, and so by Theorem (3.3), the sequence $\left\{x_{n}\right\}$ given by $x_{n+1}=$ $(1-\lambda) x_{n}+\lambda \cdot \frac{1}{x_{n}}, \quad n=0,1,2 \ldots \ldots$ converges strongly to the fixed point $p=1$ of $T$ for all values of $\lambda$ in the interval $\left(0, \frac{1}{16}\right)$.

In order to compare two fixed point iteration procedures $\left\{u_{n}\right\}_{n=0}^{\infty}$ and $\left\{v_{n}\right\}_{n=0}^{\infty}$ that converges to a certain fixed point $p$ of a given operator $T$, Rhoades [8] considered that $\left\{u_{n}\right\}$ is better than $\left\{v_{n}\right\}$ if

$$
\left\|u_{n}-p\right\| \leq\left\|v_{n}-p\right\|, \forall n
$$

Definition 8. [5] Let $\left\{a_{n}\right\}_{n=0}^{\infty}$ and $\left\{b_{n}\right\}_{n=0}^{\infty}$ be two sequences of real numbers that converges to a and $b$, respectively, and assume that there exists

$$
l=\lim _{n \rightarrow \infty}\left|\frac{a_{n}-a}{b_{n}-b}\right| .
$$


(a) If $l=0$, then it can be said that $\left\{a_{n}\right\}_{n=0}^{\infty}$ converges faster to a than $\left\{b_{n}\right\}_{n=0}^{\infty}$ to $b$;

(b) If $0<l<\infty$, then it can be said that $\left\{a_{n}\right\}_{n=0}^{\infty}$ and $\left\{b_{n}\right\}_{n=0}^{\infty}$ have the same rate of convergence.

Remark.

(i) In the case (a) we use the notation $a_{n}-a=o\left(b_{n}-b\right)$;

(ii) if $l=\infty$, then the sequence $\left\{b_{n}\right\}_{n=0}^{\infty}$ converges faster than $\left\{a_{n}\right\}_{n=0}^{\infty}$, that is, $b_{n}-n=o\left(a_{n}-a\right)$.

Suppose that for two fixed iterations procedures $\left\{u_{n}\right\}_{n=0}^{\infty}$ and $\left\{v_{n}\right\}_{n=0}^{\infty}$ both converging to the same fixed point $p$, the error estimates

$$
\begin{gathered}
\left\|u_{n}-p\right\| \leq a_{n}, \quad n=0,1,2 \ldots \\
\left\|v_{n}-p\right\| \leq b_{n}, \quad n=0,1,2 \ldots
\end{gathered}
$$

are available, where $\left\{a_{n}\right\}_{n=0}^{\infty}$ and $\left\{b_{n}\right\}_{n=0}^{\infty}$ are two sequences of positive numbers (converging to zero). Then, in view of Definition (3.6), we will adopt the following concepts.

Definition 9. [5] Let $\left\{u_{n}\right\}_{n=0}^{\infty}$ and $\left\{v_{n}\right\}_{n=0}^{\infty}$ be two fixed point iteration procedures that converge to the same fixed point $p$ and satisfy (3.10) and (3.11), respectively. If $\left\{a_{n}\right\}_{n=0}^{\infty}$ converges faster than $\left\{b_{n}\right\}_{n=0}^{\infty}$ then it can be said that $\left\{u_{n}\right\}_{n=0}^{\infty}$ converges faster than $\left\{v_{n}\right\}_{n=0}^{\infty}$ to $p$.

Example 4. [3] If we take $p=0, u_{n}=\frac{1}{(n+1)}, v_{n}=\frac{1}{n}, n \geq 1$, then $\left\{u_{n}\right\}$ is better than $\left\{v_{n}\right\}$, but $\left\{u_{n}\right\}$ does not converge faster than $\left\{v_{n}\right\}$. Indeed we have

$$
\lim _{n \rightarrow \infty} \frac{u_{n}}{v_{n}}=1
$$

and hence $\left\{u_{n}\right\}$ and $\left\{v_{n}\right\}$ have the same rate of convergence.

The example (3.9) shows that Definition (3.8) introduces a sharper concept of rate of convergence than the one considered by Rhoades [8].

In the next section we begin by proving the Browder-Gohde-Kirk fixed point theorem, which is a basic fixed point existence result for non-expansive operators. The proof will be given in a Hilbert space setting, suitable to many convergence theorems for the Krasnoselskij iterations.

\section{The main result}

Now, we are in position to state the main convergence theorems for Krasnoselskij iteration.

Theorem 3. Let $C$ be a closed bounded convex subset of a Hilbert space $H$ and $T: C \rightarrow C$ be a non-expansive operator. Then $T$ has at least one fixed point.

Proof. For fixed element $y_{0}$ in $C$ and a number $s$ with $0<s<1$, we denote

$$
T_{s}(x)=(1-s) y_{0}+s T x, \quad x \in C \text {. }
$$

Since $C$ is convex and closed, we deduce that $T_{s}: C \rightarrow C$ is a $s$-contraction and it has a unique fixed point, say $u_{s}$ (from the contraction mapping principle). On the other hand, since $C$ is closed, convex and bounded in a Hilbert space $H$, it is weakly compact. Hence we find a sequence $\left\{s_{j}\right\}$ in $(0,1)$ such that $s_{j} \rightarrow 1$ as $j \rightarrow \infty$ and $u_{j}=u_{s_{j}}$, converges weakly to 
an element $p$ of $H$. Since $C$ is weakly closed, $p$ lies in $C$. We shall prove that $p$ is a fixed point of $T$. If $u$ is any arbitrary point in $H$, we have $\left\|u_{j}-u\right\|^{2}=\left\|\left(u_{j}-p\right)+(p-u)\right\|^{2}=\left\|u_{j}-p\right\|^{2}+\|p-u\|^{2}+2\left\langle u_{j}-p, p-u\right\rangle$,

$$
\text { where } 2\left\langle u_{j}-p, p-u\right\rangle \rightarrow 0 \text { as } j \rightarrow \infty \text {, }
$$

since $u_{j}-p$ converges weakly to zero in $H$. Letting $u=T p$ above, we get

$$
\lim _{j \rightarrow \infty}\left(\left\|u_{j}-T p\right\|^{2}-\left\|u_{j}-p\right\|^{2}\right)=\|p-T p\|^{2} .
$$

Moreover, since $s_{j} \rightarrow 1$ and $T_{s_{j}} u_{j}=u_{j}$, we have

$$
\begin{aligned}
T u_{j}-u_{j} & =\left[s_{j} T u_{j}+\left(1-s_{j}\right) y_{0}\right]-u_{j}+\left(1-s_{j}\right)\left[T u_{j}-y_{0}\right] \\
& =\left(T_{s_{j}} u_{j}-u_{j}\right)+\left(1-s_{j}\right)\left(T u_{j}-y_{0}\right) \\
& =0+\left(1-s_{j}\right)\left(T u_{j}-y_{0}\right) \rightarrow 0 \text { as } j \rightarrow \infty
\end{aligned}
$$

and therefore $\lim _{j \rightarrow \infty}\left\|T u_{j}-u_{j}\right\|=0$.

On the other hand, since $T$ is non-expansive, we have

$$
\left\|T u_{j}-T p\right\| \leq\left\|u_{j}-p\right\|
$$

and hence

$$
\left\|u_{j}-T p\right\| \leq\left\|u_{j}-T u_{j}\right\|+\left\|T u_{j}-T p\right\| \leq\left\|u_{j}-T u_{j}\right\|+\left\|u_{j}-p\right\| .
$$

Thus $\lim \sup \left(\left\|u_{j}-T p\right\|-\left\|u_{j}-p\right\|\right) \leq \lim _{j \rightarrow \infty}\left\|u_{j}-T u_{j}\right\|=0$, and due to the boundedness of $C$, we have also

$$
\text { limsup }\left(\left\|u_{j}-T p\right\|^{2}-\left\|u_{j}-p\right\|^{2}\right)=\lim \sup \left(\left\|u_{j}-T p\right\|-\left\|u_{j}-p\right\|\right)\left(\left\|u_{j}-T p\right\|+\left\|u_{j}-p\right\|\right) \leq 0,
$$

which yields

$$
\lim _{j \rightarrow \infty}\left(\left\|u_{j}-T p\right\|^{2}-\left\|u_{j}-p\right\|^{2}\right)=0
$$

and hence

$$
\|p-T p\|^{2}=0
$$

that is, $p$ is a fixed point of $T$.

We can now consider a result on approximating fixed points of non-expansive mappings by means of the Krasnoselskij iteration. To this end, we start with the following concept:

Definition 10. Let $H$ be a Hilbert space and $C$ a subset of $H$. A mapping $T: C \rightarrow H$ is called demicompact if it has the property that whenever $\left\{x_{n}\right\}$ is a bounded sequence in $H$ and $\left\{T x_{n}-x_{n}\right\}$ is strongly convergent, then there exists a subsequence $\left\{x_{n_{k}}\right\}$ of $\left\{x_{n}\right\}$ which is strongly convergent.

Theorem 4. Let $C$ be a bounded closed convex subset of a Hilbert space $H$ and $T: C \rightarrow C$ be a non-expansive and demicompact operator.

(i) Then the set $F_{T}$ of fixed points of $T$ is a nonempty convex set. 
(ii) For any given $x_{0}$ in $C$ and for any fixed number $\lambda$ with $0<\lambda<1$, the Krasnoselskij iteration $\left\{x_{n}\right\}_{n=0}^{\infty}$ given by

$$
x_{n+1}=(1-\lambda) x_{n}+\lambda T x_{n}, \quad n=0,1,2 \ldots
$$

converges strongly to a fixed point of $T$.

(iii) If $x_{0} \in H$ and $\left\{x_{n}\right\}_{n=0}^{\infty}=M_{n}\left(x_{0}, \alpha_{n}, T\right)$ is the Mann iteration such that the sequence $\left\{\alpha_{n}\right\}_{n=0}^{\infty}$ is bounded away from 0 and 1 , then each of the sequence $\left\{x_{n+1}-x_{n}\right\}$ and $\left\{T x_{n}-x_{n}\right\}$ converges strongly to $0 \in H$.

\section{Proof.}

(i) Since $T$ is nonexpansive, so by theorem (4.1), $T$ has fixed points in $C$, that is, $F_{T} \neq \phi$. Further, $F_{T}$ is convex. i.e., when $x, y \in F_{T}$ and $\lambda \in[0,1]$ we have

$$
x_{\lambda}=(1-\lambda) x+\lambda y \in F_{T}
$$

Indeed, $\left\|T x_{\lambda}-x\right\|=\left\|T x_{\lambda}-T x\right\| \leq\left\|x_{\lambda}-x\right\|$ and $\left\|T x_{\lambda}-y\right\| \leq\left\|x_{\lambda}-y\right\|$,

$$
\begin{aligned}
\Rightarrow\|x-y\| & \leq\left\|x-T x_{\lambda}+T x_{\lambda}-y\right\| \\
& \leq\left\|x-T x_{\lambda}\right\|+\left\|T x_{\lambda}-y\right\| \\
& \leq\|x-y\| .
\end{aligned}
$$

This shows that for some $a, b$ with $0 \leq a, b \leq 1$, we have $x-T x_{\lambda}=a\left(x-x_{\lambda}\right)$ and $y-T x_{\lambda}=b\left(y-x_{\lambda}\right)$ from which it follows that $T x_{\lambda}=x_{\lambda} \in F_{T}$.

(ii) For any $x_{0}$ in $C$, the sequence $\left\{x_{n}\right\}_{n=0}^{\infty}$ given by (4.1) lies in $C$ and is bounded. Let $p$ be a fixed point of $T$, and so of averaged map $T_{\lambda}$, given by

$$
T_{\lambda}=(1-\lambda) I+\lambda I, \quad(I=\text { identity operator })
$$

We first prove that the sequence $\left\{x_{n}-T x_{n}\right\}_{n \in \mathbb{N}}$ converges strongly to zero. Indeed $x_{n+1}-p=(1-\lambda) x_{n}+\lambda T x_{n}-p=(1-\lambda)\left(x_{n}-p\right)+\lambda\left(T x_{n}-p\right)$.

On the other hand, for any constant $a$,

$$
a\left(x_{n}-T x_{n}\right)=a\left(x_{n}-p\right)-a\left(T x_{n}-p\right) .
$$

Then $\left\|x_{n+1}-p\right\|^{2}=(1-\lambda)^{2}\left\|x_{n}-p\right\|^{2}+\lambda^{2}\left\|T x_{n}-p\right\|^{2}+2 \lambda(1-\lambda)\left\langle T x_{n}-p, x_{n}-p\right\rangle$

$$
\text { and } \left.a^{2}\left\|x_{n}-T x_{n}\right\|^{2}=a^{2}\left\|x_{n}-p\right\|^{2}+a^{2}\left\|T x_{n}-p\right\|^{2}-2 a^{2}\left\langle T x_{n}-p, x_{n}-p\right\rangle\right\rangle \text {. }
$$

Hence, adding the corresponding sides of the preceding two inequalities and using the fact that $T$ is nonexpansive and $T_{p}=p$, we get

$$
\left\|x_{n+1}-p\right\|^{2}+a^{2}\left\|x_{n}-T x_{n}\right\|^{2} \leq\left[2 a^{2}+\lambda^{2}+(1-\lambda)^{2}\right] \cdot\left\|x_{n}-p\right\|^{2}+2\left[\lambda(1-\lambda)-a^{2}\right] \cdot\left\langle T x_{n}-p, x_{n}-p\right\rangle .
$$

If we choose now an $a$ such that $a^{2} \leq \lambda(1-\lambda)$, then from the last inequality we have

$$
\left\|x_{n+1}-p\right\|^{2}+a^{2}\left\|x_{n}-T x_{n}\right\|^{2} \leq\left(2 a^{2}+\lambda^{2}+(1-\lambda)^{2}-2 \lambda(1-\lambda)-2 a^{2}\right)\left\|x_{n}-p\right\|^{2}=\left\|x_{n}-p\right\|^{2}
$$


(by using the Cauchy Schwarz inequality, $\left\langle T x_{n}-p, x_{n}-p\right\rangle \leq\left\|T x_{n}-p\right\| \cdot\left\|x_{n}-p\right\| \leq\left\|x_{n}-p\right\|^{2}$ ).

Let $a^{2}=\lambda(1-\lambda)>0$ and summing up the obtained inequality

$$
a^{2}\left\|x_{n}-T x_{n}\right\|^{2} \leq\left\|x_{n}-p\right\|^{2}-\left\|x_{n+1}-p\right\|^{2}
$$

for $n=0$ to $n=N$ we obtain

$$
\begin{aligned}
\lambda(1-\lambda) \sum_{n=0}^{N}\left\|x_{n}-T x_{n}\right\|^{2} & \leq \sum_{n=0}^{N}\left[\left\|x_{n}-p\right\|^{2}-\left\|x_{n+1}-p\right\|^{2}\right] \\
& =\left\|x_{0}-p\right\|^{2}-\left\|x_{N+1}-p\right\|^{2} \\
& \leq\left\|x_{0}-p\right\|^{2}
\end{aligned}
$$

which shows that $\sum_{n=0}^{\infty}\left\|x_{n}-T x_{n}\right\|^{2}<\infty$ and hence $\left\|x_{n}-T x_{n}\right\| \rightarrow 0$, as $n \rightarrow \infty$. As $T$ is demicompact, it follows that there exists a strongly convergent subsequence $\left\{x_{n_{i}}\right\}$ such that $x_{n_{i}} \rightarrow p \in F_{T}$. Since $T$ is nonexpansive, $T x_{n_{i}} \rightarrow T p$ and $T p=p$. This shows that the convergence of the entire sequence $\left\{x_{n}\right\}_{n=0}^{\infty}$ to $p$ and now follows from the inequality $\left\|x_{n+1}-p\right\| \leq\left\|x_{n}-p\right\|$, which can be deduce from the nonexpansiveness of $T$ and is valid for each $n$.

(iii) For $x_{0} \in H$ and $M_{n}\left(x_{0}, \alpha_{n}, T\right)$ is a Mann iteration such that

$$
\left\|x_{{ }^{\prime} n+1}-x_{n}\right\|=\alpha_{n}\left\|T x_{n}-x_{n}\right\|,
$$

and hence, having in view that $0<a \leq \alpha_{n} \leq b<1$, if either one of the sequences $\left\{x_{n+1}-x_{n}\right\}$ or $\left\{T x_{n}-x_{n}\right\}$ converges to 0 then the other does also. If $\lim \left\|x_{n}-p\right\|=0$, then obviously $\lim \left\|x_{n+1}-x_{n}\right\|=0$. Otherwise, since the sequence $\left(\left\|x_{n}-p\right\|\right)$ is non-increasing, we certainly have $\lim \left\|x_{n}-p\right\|=d>0$. We define now the sequences $\left\{w_{n}\right\},\left\{y_{n}\right\}$ and $\left\{z_{n}\right\}$ by

$$
w_{n}=\left(x_{n}-p\right) /\left\|x_{n}-p\right\|, y_{n}=\left(T x_{n}-p\right) /\left\|x_{n}-p\right\|, \text { and, respectively } z_{n}=\left(x_{n+1}-p\right) /\left\|x_{n}-p\right\| .
$$

Since we have that

$$
x_{n+1}-p=\left(1-\alpha_{n}\right)\left(x_{n}-p\right)+\alpha_{n}\left(T x_{n}-p\right)
$$

by dividing it by $\left\|x_{n}-p\right\|$ it results that

$$
z_{n}=\left(1-\alpha_{n}\right) w_{n}+\alpha_{n} y_{n}
$$

Since $\left\|w_{n}\right\|=1,\left\|y_{n}\right\| \leq 1$ for all $n$, and if $\lim \left\|z_{n}\right\|=1$, then $\lim \left\|w_{n}-y_{n}\right\|=0$, which implies that $\left\|z_{n}\right\| \rightarrow d / d=1$, we have that $\lim \left\|w_{n}-y_{n}\right\|=0$, which gives $\lim \left\|T x_{n}-x_{n}\right\|=0$, and this completes the proof.

\section{Remark.}

(i) From the proof of the theorem (4.3) it shows that $T_{\lambda}$ given by (4.2) is asymptotically regular, i.e., $\left\|T_{\lambda}^{n} x-T_{\lambda}^{n+1} x\right\| \rightarrow$ 0 , as $n \rightarrow \infty$, for any $x \in C$, that is, $x_{n}-x_{n+1} \rightarrow 0$, as $n \rightarrow \infty$, for any $x_{0} \in C$. The existence of the previous limit alone does not imply genearlly the convergence of the sequence $\left\{x_{n}\right\}_{n=0}^{\infty}$ to fixed point of $T$ as there is one more additional assumption of demicompactness of $T$ in theorem (4.3). There are other possible additional assumptions to ensure the convergence of $\left\{x_{n}\right\}_{n=0}^{\infty}$ under the hypothesis of asymptotic regularity. For example, in the case of the real line, $C=[a, b]$ the closed and bounded interval and $T: C \rightarrow C$ a continuous function, Hilam [7] showed that the Picard iteration associated to $T$ converges if and only if it is asymptotically regular; 
(ii) The Krasnoselskij iteration is in fact the Picard iteration corresponding to the averaged operator $T_{\lambda}$ associated to $T$ and defined by (4.2).

If in theorem (4.3) we remove the assumption that $T$ is demicompact, then the Krasnoselskij iteration does not longer converge strongly, in general, but it converges at least weakly to a fixed point, which is shown by the next theorem (4.5).

Theorem 5. Let $C$ be a bounded closed convex subset of a Hilbert space $H$ and $T: C \rightarrow C$ be a non-expansive and operator. Then, for any $x_{0}$ in $C$, the Krasnoselskij iteration $\left\{x_{n}\right\}_{n=0}^{\infty}$ given by

$$
x_{n+1}=(1-\lambda) x_{n}+\lambda T x_{n}, \quad n=0,1,2 \ldots
$$

converges weakly to a fixed point of $T$.

Proof. Let $F_{T}$ be the set of all fixed points of $T$ in $C$. Since $T$ is nonexpansive, for each $p \in F_{T}$ and each $n$ we have

$$
\left\|x_{n+1}-p\right\| \leq\left\|x_{n}-p\right\|
$$

which shows that the function $g(p)=\lim _{n \rightarrow \infty}\left\|x_{n}-p\right\|$ is well defined and is a lower semicontinuous convex function on $F_{T}$. Let

$$
d_{0}=\inf \left\{g(p): p \in F_{T}\right\}
$$

For each $\varepsilon>0$, the set

$$
F_{\varepsilon}=\left\{y: g(y) \leq d_{0}+\varepsilon\right\}
$$

is closed, convex, nonempty and bounded and hence, weakly compact. Therefore $\cap_{\varepsilon>0} F_{\varepsilon} \neq 0$, and in fact

$$
\cap_{\varepsilon>0} F_{\varepsilon}=\left\{y: g(y)=d_{0}\right\} \equiv F_{0} .
$$

Since $F_{0}$ contains exactly one point. Indeed, since $F_{0}$ is convex and closed, for $p_{0}, p_{1} \in F_{0}$, and $p \lambda=(1-\lambda) p_{0}+\lambda p_{1}$,

$$
\begin{aligned}
g^{2}(p \lambda) & =\lim _{n \rightarrow \infty}\left\|p \lambda-x_{n}\right\|^{2}=\lim _{n \rightarrow \infty}\left(\left\|\lambda\left(p_{1}-x_{n}\right)+(1-\lambda)\left(p_{0}-x_{n}\right)\right\|^{2}\right) \\
& =\lim _{n \rightarrow \infty}\left(\lambda^{2}\left\|p_{1}-x_{n}\right\|^{2}+(1-\lambda)^{2}\left\|p_{0}-x_{n}\right\|^{2}+2 \lambda(1-\lambda)\left\langle p_{1}-x_{n}, p_{0}-x_{n}\right\rangle\right) \\
& =\lim _{n \rightarrow \infty}\left(\lambda^{2}\left\|p_{1}-x_{n}\right\|^{2}+(1-\lambda)^{2}\left\|p_{0}-x_{n}\right\|^{2}+2 \lambda(1-\lambda)\left\|p_{1}-x_{n}\right\| \cdot\left\|p_{0}-x_{n}\right\|\right) \\
& +\lim _{n \rightarrow \infty}\left\{2 \lambda(1-\lambda)\left[\left\langle p_{1}-x_{n}, p_{0}-x_{n}\right\rangle-\left\|p_{1}-x_{n}\right\| \cdot\left\|p_{0}-x_{n}\right\|\right]\right\} \\
& =g^{2}(p)+\lim _{n \rightarrow \infty}\left\{2 \lambda(1-\lambda)\left[\left\langle p_{1}-x_{n}, p_{0}-x_{n}\right\rangle-\left\|p_{1}-x_{n}\right\| \cdot\left\|p_{0}-x_{n}\right\|\right]\right\} .
\end{aligned}
$$

Hence $\lim _{n \rightarrow \infty}\left\{2 \lambda(1-\lambda)\left[\left\langle p_{1}-x_{n}, p_{0}-x_{n}\right\rangle-\left\|p_{1}-x_{n}\right\| \cdot\left\|p_{0}-x_{n}\right\|\right]\right\}=0$.

Since $\left\|p_{1}-x_{n}\right\| \rightarrow d_{0}$ and $\left\|p_{0}-x_{n}\right\| \rightarrow d_{0}$, the latter relation implies that

$$
\begin{aligned}
\left\|p_{1}-p_{0}\right\|^{2} & =\left\|\left(p_{1}-x_{n}\right)+\left(x_{n}-p_{0}\right)\right\|^{2} \\
& =\left\|p_{1}-x_{n}\right\|^{2}+\left\|x_{n}-p_{o}\right\|^{2}-2\left\langle p_{1}-x_{n}, p_{0}-x_{n}\right\rangle \\
& \rightarrow d_{0}^{2}-d_{0}^{2}-2 d_{0}^{2}=0
\end{aligned}
$$

giving a contradiction.

Now in order to show that $x_{n}=U_{\lambda}^{n} x_{0} \rightarrow p_{0}$, is suffices to assume that $x_{n_{j}} \rightarrow p$ for an infinite subsequence and then prove 
that $p=p_{0}$. Since $p \in F_{T}$ and considering the definition of $g$ and the fact that $x_{n_{j}} \rightarrow p$, we have

$$
\begin{aligned}
\left\|x_{n}-p_{0}\right\|^{2} & =\left\|x_{n_{j}}-p+p-p_{0}\right\|^{2} \\
& =\left\|x_{n_{j}}-p\right\|+\left\|p-p_{0}\right\|^{2}-2\left\langle x_{n_{j}}-p, p-p_{0}\right\rangle \\
& \rightarrow g^{2}(p)+\left\|p-p_{0}\right\|^{2}=g^{2}\left(p_{0}\right)=d_{0}^{2} .
\end{aligned}
$$

Since $g^{2}(p) \geq d_{0}^{2}$, the last inequality implies that

$$
\left\|p-p_{0}\right\| \leq 0
$$

which implies that $p=p_{0}$.

We present here convergence theorem for the Krasnoselskij iteration scheme in the class of generalized pseudocontractive operators which are also Lipschitzian.

Theorem 6. Let $H$ be a real Hilbert space and $C$ a nonempty closed convex subset of $H$. Let $T: C \rightarrow C$ be a Lipschitzian generalized pseudocontractive operator with corresponding constants $s \geq 1$ and $0<r<1$. Then

(i) $T$ has a unique fixed point $p$ in $C$;

(ii) For any $x_{0} \in C$ and $\lambda \in(0, a)$ with' $a^{\prime}$ given by (3.6), the Krasnoselskij iteration $\left\{x_{n}\right\}_{n=0}^{\infty}=K_{n}\left(x_{0}, \lambda, T\right)$ converges strongly to $p$;

(iii) For any $x_{0} \in C$ and $\left\{\alpha_{n}\right\}_{n=0}^{\infty}$ in $[0,1]$ satisfying (3.7), the Mann iteration $\left\{x_{n}\right\}_{n=0}^{\infty}=M_{n}\left(x_{0}, \alpha_{n}, T\right)$ converges strongly to $p$;

(iv) For any Mann iteration converging to $p$, with $0 \leq \alpha_{n} \leq b<1$, there exists Krasnoselskij that converges faster to $p$.

Proof. For all $\lambda \in[0,1]$, consider the operator $T_{\lambda}$ on $C$ given by

$$
T_{\lambda} x=(1-\lambda) x+\lambda T x, \quad x \in C
$$

since $C$ is convex, we have $T_{\lambda}(C) \subset C$, for all $\lambda \in[0,1]$. From the generalized pseudocontractive and Lipschitzian conditions on $T$ and

$$
\begin{aligned}
&\left\|T_{\lambda} x-T_{\lambda} y\right\|^{2}=\|(1-\lambda)(x-y)+\lambda(T x-T y)\|^{2} \\
&=(1-\lambda)^{2}\|x-y\|^{2}+2 \lambda(1-\lambda)\langle T x-T y, x-y\rangle+\lambda^{2}\|T x-T y\|^{2} \\
&\left\|T_{\lambda} x-T_{\lambda} y\right\|^{2} \leq\left[(1-\lambda)^{2}+2 \lambda(1-\lambda) r+\lambda^{2} s^{2}\right]\|x-y\|^{2}, \\
& \text { so }\left\|T_{\lambda} x-T_{\lambda} y\right\| \leq \theta\|x-y\|, \text { for all } x, y \text { in } C,
\end{aligned}
$$

where $0<\theta=\left[(1-\lambda)^{2}+2 \lambda(1-\lambda) r+\lambda^{2} s^{2}\right]^{1 / 2}$, as $\lambda<a$.

Since $C$ is a closed subset of a Hilbert space, $C$ is a complete metric space. Then by Banach contraction principle, $T_{\lambda}$ has a unique fixed point say, $q$ in $C$ and the Picard iteration associated to $T_{\lambda}$,

$$
x_{n+1}=T_{\lambda} x_{n}, \quad n \geq 0
$$

converges strongly to $q$, for any $x_{0} \in C$. By using theorem (4.3), $\left\{x_{n}\right\}_{n=0}^{\infty}$ is exactly the Krasnoselskij iteration $K_{n}\left(x_{0}, \lambda, T\right)$ associated to $T$ and the fact that $F(T)=F\left(T_{\lambda}\right)$, for all $\lambda \in(0,1)$, that is, $p=q$ is the unique fixed point of 
$T$, so we get (i) and (ii).

(iii) Let $\left\{x_{n}\right\}_{n=0}^{\infty}$ be the Mann iteration with $\left\{\alpha_{n}\right\}_{n=0}^{\infty} \subset[0,1]$ satisfying (3.7), consider $t, 0<t<1$, denote $a_{n}=\frac{1}{t} \alpha_{n}, n=0,1,2, \ldots \ldots$. Then the Mann iteration will be given by $x_{n+1}=\left(1-t a_{n}\right) x_{n}+t a_{n} T x_{n}, n=0,1,2, \ldots \ldots \ldots$, we have

$$
\begin{gathered}
\left\|x_{n+1}-p\right\|=\left\|\left(1-a_{n}\right) x_{n}+a_{n}\left[(1-t) x_{n}+t T x_{n}\right]-p\right\| \\
\left\|x_{n+1}-p\right\| \leq\left(1-a_{n}\right)\left\|x_{n}-p\right\|+a_{n}\left\|(1-t)\left(x_{n}-p\right)+t\left(T x_{n}-T p\right)\right\| .
\end{gathered}
$$

using the properties of $T$ we find that

$$
\begin{aligned}
\left\|t\left(T x_{n}-T p\right)+(1-t)\left(x_{n}-p\right)\right\|^{2} & =(1-t)^{2}\left\|x_{n}-p\right\|^{2}+2 t(1-t)\left\langle T x_{n}-p, x_{n}-p\right\rangle+t^{2}\|T x-p\|^{2} \\
& \leq(1-t)^{2}\left\|x_{n}-p\right\|^{2}+2 t(1-t) r\left\|x_{n}-p\right\|^{2}+t^{2} s^{2}\left\|x_{n}-p\right\|^{2} \\
& =\left[(1-t)^{2}+2 t(1-t) r+t^{2} s^{2}\right]\left\|x_{n}-p\right\|^{2}
\end{aligned}
$$

By (4.6) and (4.7) we get

$$
\begin{gathered}
\left\|x_{n+1}-p\right\| \leq\left\{1-a_{n}+a_{n}\left[(1-t)^{2}+2 t(1-t) r+t^{2} s^{2}\right]^{1 / 2}\right\}\left\|x_{n}-p\right\| \\
=\left(1-(1-\theta) a_{n}\right)\left\|x_{n}-p\right\| \\
\leq \prod_{k=1}^{n}\left(1-(1-\theta) a_{k}\right)\left\|x_{1}-p\right\|
\end{gathered}
$$

where $0 \leq \theta=\left[(1-t)^{2}+2 t(1-t) r+t^{2} s^{2}\right]^{1 / 2}<1$, for all $t$ such that $0<t<2(1-r) /\left(1-2 r+s^{2}\right)$.

Since by (3.7), $\sum_{n=0}^{\infty} \alpha_{n}$ diverges, it follows that $\sum_{n=0}^{\infty} a_{n}$ diverges too, and in view of $\theta<1$ we get

$$
\lim _{n \rightarrow \infty} \prod_{k=1}^{n}\left(1-(1-\theta) a_{k}\right)=0
$$

so by (4.8) shows that $\left\{x_{n}\right\}$ converges strongly to $p$.

(iv) Take $x=x_{n}, y=x_{n-1}$ in (4.4) we obtain $\left\|x_{n+1}-x_{n}\right\| \leq \theta\left\|x_{n}-x_{n-1}\right\|$. By induction, which yields

$$
\left\|x_{n+1}-x_{n}\right\| \leq \theta^{n}\left\|x_{1}-x_{0}\right\|
$$

and hence by triangle rule we get

$$
\left\|x_{n+p}-x_{n}\right\| \leq \theta^{n}\left(1+\theta+\ldots+\theta^{p-1}\right)\left\|x_{1}-x_{0}\right\|
$$


valid for all $n, p \in \mathbb{N}$. Now letting $p \rightarrow \infty$ in (4.9) and by using part (ii), we have

$$
\left\|x_{n}-x\right\|=\frac{\theta^{n}}{1-\theta}\left\|x_{1}-x_{0}\right\| .
$$

therefore, in view of (4.8) and (4.10), in order to compare Krasnoselskij and Mann iterations, we have to compare

$$
\theta^{n} \text { and } \prod_{k=1}^{n}\left[1-(1-\theta) a_{k}\right]
$$

Let $\left\{x_{n}\right\}_{n=0}^{\infty}$ be a certain Maan iteration converging to $p$, with $\left\{\alpha_{n}\right\}_{n=0}^{\infty}$ satisfying $0 \leq \alpha_{n} \leq b<1$. Then $a_{k} \leq b / t$ (denote $b / t$ by $b)$ and for any $m, 0<m<1$, we find $\theta \in(0,1)$ such that

$$
b<\frac{1-(\theta / m)}{1-\theta}
$$

clearly, which implies that

$$
\theta<\frac{m(1-b)}{1-b m}
$$

using the fact that $a_{k} \leq b$, it gives

$$
\frac{\theta}{1-(1-\theta) a_{k}} \leq m<1
$$

which shows that

$$
\frac{\theta}{\prod_{k=1}^{n}\left[1-(1-\theta) a_{k}\right]} \leq \lim _{n \rightarrow \infty} m^{n}=0
$$

so the Krasnoselskij iteration $\left\{x_{n}\right\}_{n=0}^{\infty}=K_{n}\left(x_{0}, \quad \theta, \quad T\right)$ converges faster than the Mann iteration $\left\{x_{n}\right\}_{n=0}^{\infty}=M_{n}\left(x_{0}, \alpha_{n}, T\right)$.

In order to complete the proof, we still need to show that the intervals $(0, a)$ with $a$ given by (3.6) and $\left(0, \frac{m(1-b)}{1-m}\right)$ have a nonempty intersection. But this is immediate, since $\frac{m(1-b)}{1-m}>0$ and $0<a=\frac{2(1-r)}{1-2 r+s^{2}} \leq 1$, under the hypothesis of the theorem.

Remark.(i) Part (iv) of theorem (4.6) shows that, in order to approximate the fixed points of a Lipschitzian and pseudocontractive operator $T$, it is always more convenient to use a certain Krasnoselskij iteration in the family (3.2) with $\lambda \in(0, a)$ and $^{\prime} a$ ' is given by (3.6).

\section{Competing interests}

The authors declare that they have no competing interests.

\section{Authors' contributions}

All authors have contributed to all parts of the article. All authors read and approved the final manuscript. 


\section{References}

[1] G. V. R. Babu and K. N. V. Vara Prashad, Mann iteration converges faster than Ishikawa iteration for the class of Zamfirescu operators, Fixed Point Theory and applications Vol. 2006, article ID 49615, Pages 16 DOI 10.1155/FPTA/2006/49615.

[2] V. Berinde, Approximating fixed points of Lipschitzian pseudocontractions, in Mathematics and mathematics education (Bethlehem, 2000), World Sci. Publishing, River Edge, 2002, pp. 73-81.

[3] V. Berinde, Comparing Krasnoselskij and Mann iterations for Lipschitzian and generalized pseudocontractive operators, Fixed Point Theory and Applications, (Valencia, 2003), Yokohama publishers, Yokohama, 2004.

[4] V. Berinde, Iterative Approximation of Fixed Points, Editura Efemeride, Baia Mare, (2002).

[5] V. Berinde, Picard iteration converges faster than the Mann iteration for the class of qausicontractive operators, Fixed Point Theory and Application 2004, No. 2, 97-105.

[6] F. E. Browder and W. V. Petryshyn, Construction of fixed points of nonlinear mappings in Hilbert spaces J. Math. Anal. Appl. 20 (1967), 197-228.

[7] B. P. Hillam, A characterization of the convergence of successive approximations. Amer. Math. Monthly, 83. No. 4, 273 (1976).

[8] B. E. Rhoades, Comments on two fixed point iteration methods J. Math. Anal. Appl. 56 (1976), No. 2, 741-750.

[9] R. U. Verma, A fixed-point theorem involving Lipschitzian generalized pseudocontractions Proc. Roy. Irish Acad. sect. A 97 (1997), No. 1, 83-86.

[10] T. Zamfirescu, Fix point theorems in metric spaces, Arch. Math. (Basel), 23 (1972), 292-298. 